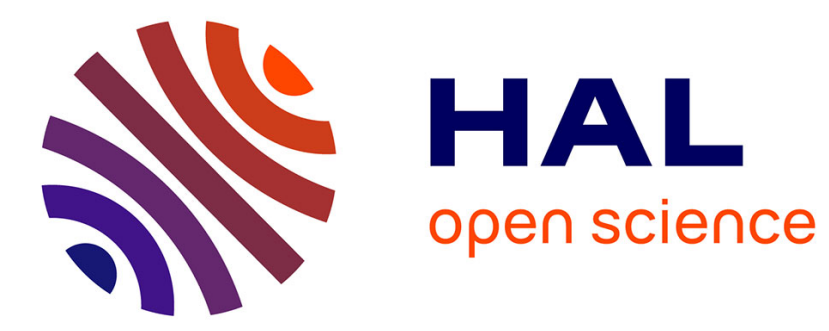

\title{
Sensing coupling paths in an equipment
}

Andrea Cozza, Charlie Galle, Jean-Pierre Brasile, Christian Carel

\section{To cite this version:}

Andrea Cozza, Charlie Galle, Jean-Pierre Brasile, Christian Carel. Sensing coupling paths in an equipment. 2012 Asia-Pacific Symposium on Electromagnetic Compatibility, May 2012, Singapore, Singapore. pp.TH-PM-RC-6. hal-00700944

\section{HAL Id: hal-00700944 https://hal.science/hal-00700944}

Submitted on 24 May 2012

HAL is a multi-disciplinary open access archive for the deposit and dissemination of scientific research documents, whether they are published or not. The documents may come from teaching and research institutions in France or abroad, or from public or private research centers.
L'archive ouverte pluridisciplinaire HAL, est destinée au dépôt et à la diffusion de documents scientifiques de niveau recherche, publiés ou non, émanant des établissements d'enseignement et de recherche français ou étrangers, des laboratoires publics ou privés. 


\title{
Sensing Coupling Paths in an Equipment
}

\author{
${ }^{1}$ Andrea Cozza, ${ }^{2}$ Charlie Galle, ${ }^{2}$ Jean-Pierre Brasile, ${ }^{2}$ Christian Carel \\ 1 Département de Recherche en Électromagnétisme \\ Laboratoire des Signaux et Systèmes (L2S), UMR8506 SUPELEC - Univ Paris-Sud - CNRS \\ 3 rue Joliot-Curie, 91192 Gif-sur-Yvette, France \\ Contact email : andrea.cozza@supelec.fr \\ 2 Thales Communications S.A. \\ 160 Boulevard de Balmy, 92700 Colombes, France
}

\begin{abstract}
We present experimental results proving that a Time-Reversal Electromagnetic Chamber allows studying the susceptibility of a device to external radiated interference. The technique here proposed is based on the generation of wavefronts focusing over an arbitrary position in space, leading to a spatial resolution of a half-wavelength. By monitoring the level of energy transmitted through the shielding of the device, it is found that this technique can identify the presence of coupling paths (e.g., slots), their position and orientation. The information thus retrieved is much richer than that yielded by standard EMC tests in anechoic or reverberation chambers, where the position of such coupling paths is not readily accessible.
\end{abstract}

Index Terms-Cavities, stochastic fields, test facilities.

\section{INTRODUCTION}

When an electrical device needs to be protected from external interferences, its shielding takes a central role in the design phase. Applications requiring an attentive shielding design range from electromagnetic compatibility (EMC) of everyday devices to the protection of sensitive equipments in electronic warfare. In order to assess the level of protection of the device, tests are typically carried out in controlled electromagnetic environments, such as anechoic and reverberation chambers (RCs). In both cases, although the type of radiated interference impinging on the device do not share the same nature, the electromagnetic stress applied to the device is meant as a way of identifying the presence of weak points in the shielding, through which interferences can couple to the electrical and electronic devices it is expected to protect.

Tests of this type can be carried out within the framework set by international standards, thus only requiring to assess the passing or not of a test. In these cases, this kind of tests are sufficient. Conversely, as soon as these are rather meant as a way of obtaining detailed information about the reasons of the failing, current test procedure are wanting. This outcome is well-known in RCs, where the complex propagation environment hinders any direct interpretation of the coupling mechanisms: in simple words, as a number of aggressions take place at the same time, it is impossible to sort out the contributions from each impinging wavefront.

At the other end of the spectrum of test facilities, anechoic chambers and in general all environments aimed at emulating free-space propagation, only provide a partially better performance. As a matter of fact, quasi-planar wavefronts are typically generated in these cases, thus submitting an extended

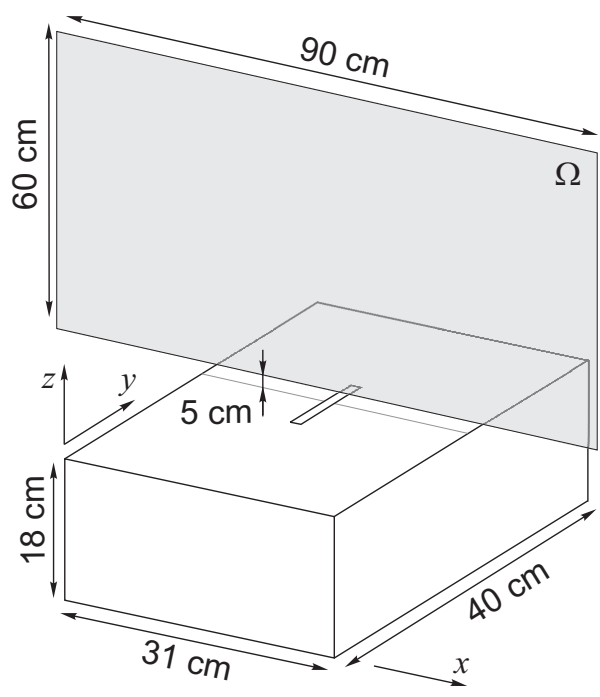

Fig. 1: A schematic representation of the experimental setup, showing the EUT and the surface over which the electric-field distributions shown in Fig. 2 were scanned.

region of the device to the impinging wavefront. Hence, if a coupling path is present at any position of the device exposed to the testing wavefront, it is not possible to find out at what position the coupling occurs and through what type of mechanism. In other words, spatial resolution is non-existent, while at least directions of arrival can be accessed; these limitations are particularly important when dealing with large devices, where visual inspection is not a viable option.

The aim of this paper is to demonstrate that the TREC (Time-Reversal Electromagnetic Chamber) technique [1] can provide a substantial improvement in this respect, by ensuring a spatial resolution only limited by diffraction phenomena [2]. By exploiting the ability of the TREC to generate arbitrary wavefronts within an RC, in particular with any position for their phase center, it is possible to use the focal spot thus created in order to sense the boundaries of the devices, and ultimately to detect the presence of apertures like slots and their orientation. 


\section{GENERATING A MOVING FOCAL SPOT}

The TREC technique was originally proposed as a way of improving the energetic performance of RCs [3], while allowing to control the polarization of the field generated within an RC [4]. The unusual features of the TREC emerge from the merging of the field-diffusive properties of RCs and the self-healing provided by phase-conjugated wavefronts [5], [6]. It is composed of an overmoded cavity where one or more antennas are found at its interior. These antennas take on the role of a time-reversal mirror (TRM), a concept introduced in acoustics and described, e.g., in [7]. Standard applications of time-reversal techniques in such a scenario would require an initial phase during which an auxiliary point-like source would generate a diverging wavefront: the signals received by the TRM antennas are recorded during this phase. Subsequently, these signals are time-reversed, i.e., phase conjugated, and applied as excitation signals to the TRM antennas: as proven in acoustics, the result of this operation is a wavefront sharing the same properties of the diverging one generated in the first place, but now converging back onto the original position of the auxiliary source. The major limitation of this procedure is the need to repeat the two phases as soon as the auxiliary source is modified (i.e., its position changed), making it an unwieldy experimental procedure, especially when dealing with hundreds of configurations.

As opposed to this state of affairs, the TREC can generate an arbitrary wavefront by means of simple signal-processing techniques. The idea is to perform a single initial characterization of the Green's functions relating the TRM antennas to the electromagnetic field observed over an auxiliary surface $\Sigma$ containing the device under test. From this knowledge, it is possible to retrieve the signals $V_{i}(\omega)$ that would have been received by the TRM antennas if a wavefront $\boldsymbol{E}_{\mathrm{wf}}(\boldsymbol{r}, \omega)$ were generated by a source within the volume defined by the auxiliary surface $\Sigma$. In particular, it can be proven that a TRM antenna assimilable to an Hertzian dipole positioned at $\boldsymbol{r}_{i}$ and oriented along the direction $\boldsymbol{q}_{i}$ would receive a signal

$$
V_{i}(\omega)=\int_{\Sigma} \boldsymbol{N}_{\mathrm{eq}, i}\left(\boldsymbol{r}^{\prime}\right) \cdot \boldsymbol{E}_{\mathrm{wf}}\left(\boldsymbol{r}^{\prime}\right) \mathrm{d} \boldsymbol{r}^{\prime}
$$

with

$$
\boldsymbol{N}_{\mathrm{eq}, i}(\boldsymbol{r})=\zeta_{0}^{-1} \tilde{\boldsymbol{G}}_{\mathrm{ee}}\left(\boldsymbol{r}, \boldsymbol{r}_{i}\right) \cdot \hat{\boldsymbol{q}}_{i}+\hat{\boldsymbol{r}} \times \tilde{\boldsymbol{G}}_{\mathrm{em}} \cdot \hat{\boldsymbol{q}}_{i}\left(\boldsymbol{r}, \boldsymbol{r}_{i}\right),
$$

$\tilde{\boldsymbol{G}}_{\mathrm{ee}}\left(\boldsymbol{r}_{1}, \boldsymbol{r}_{2}\right)$ and $\tilde{\boldsymbol{G}}_{\mathrm{em}}\left(\boldsymbol{r}_{1}, \boldsymbol{r}_{2}\right)$ being the dyadic Green's functions of the medium between an electric source (a TRM antenna) and the electric and magnetic field observed in the medium.

From this formalism it is clear that it is not necessary to have previous knowledge of Green's function over the entire space of the cavity, but it can rather be limited to the surface $\Sigma$, a direct consequence of Huygen's principle. Experimental results proving the validity of these concepts have been provided in [8].

These ideas can be applied to the problem of identifying coupling paths in a device in the following manner. Let us consider a series of wavefronts $\boldsymbol{E}_{\mathrm{wf}}(\boldsymbol{r})$ corresponding to pointlike sources with a phase center found at a generic position $\boldsymbol{r}_{0}$. Once the vector transfer functions $\boldsymbol{N}(\boldsymbol{r})$ are known, (1) allows a direct definition of the signals that should be applied to the TRM antennas in order to generate these same wavefronts focusing onto $\boldsymbol{r}_{0}$, without any need for mechanical displacements. Hence, by synthesizing the signals $V_{i}(\omega)$ and feeding their time-reversed version to the TRM antennas, a focal spot of about half the wavelength associated to the central frequency of the excitation signals can be expected to appear over $\boldsymbol{r}_{0}$ at the time of focusing.

Having this feature available in a test facility means that it is possible to expose the device to an aggression just over a portion of its surface, in such a way that a voltage or current could be transmitted and observed at its interior only in the case an aperture appeared over the focal spot, thus yielding a direct information about the position of an aperture. Moreover, its orientation, or rather its polarizability [9], can be inferred by generating a wavefront with a varying polarization: the case of maximum transmission yields a direct guess about the polarizability. Indeed, as already recalled, the TREC technique allows a fine control of the polarization of the field at the focus position [4]

\section{EXPERIMENTAL RESULTS}

These concepts were experimentally tested in Supélec's RC $\left(3.08 \times 1.84 \times 2.44 \mathrm{~m}^{3}\right)$, using a TRM composed of a single bow-tie antenna with a bandwidth of about $500 \mathrm{MHz}$ centered at $1.2 \mathrm{GHz}$, mounted perpendicularly to one of the walls of the cavity. The mock-up device was a metallic box where a longitudinal slot $15 \mathrm{~cm}$ long and $3 \mathrm{~mm}$ wide was opened, as shown in Fig. 1. By means of a planar styrofoam support positioned as in Fig. 1, the transfer functions between the TRM antenna and the electric field it would generate over the surface $\Omega$ were measured. This task was carried out by means of an optical E-field probe, manufactured by Enprobe, model EFS-105. Three transfer functions were measured for the three Cartesian field components, thanks to the linear polarization of this probe. It is therefore possible to study the field distribution generated by any signal applied to the TRM antenna without having to repeat these measurements.

Wavefronts associated to the field radiated by two-element arrays were considered, centered at different positions oriented in such a way to generate a radiation pattern with a main lobe oriented along the outward normal to the device surface. Equation (1) allows computing the signals $V_{i}(\omega)$ needed in order to generate replicas of these wavefronts focusing back at the phase center of their virtual sources. An example of results obtained is given in Fig. 2, where the phase center was moved of $12 \mathrm{~cm}$ along the top surface of the device, for pulsed signals with a bandwidth of $200 \mathrm{MHz}$ and centered around $1.2 \mathrm{GHz}$. Colors are set to the same scale for the two cases, indicating negligible differences and thus an excellent reproducibility of the aggression, a direct consequence of the self-averaging properties of time-reversed wavefronts in complex media [6]. Even the orientation of the E-field lines is 

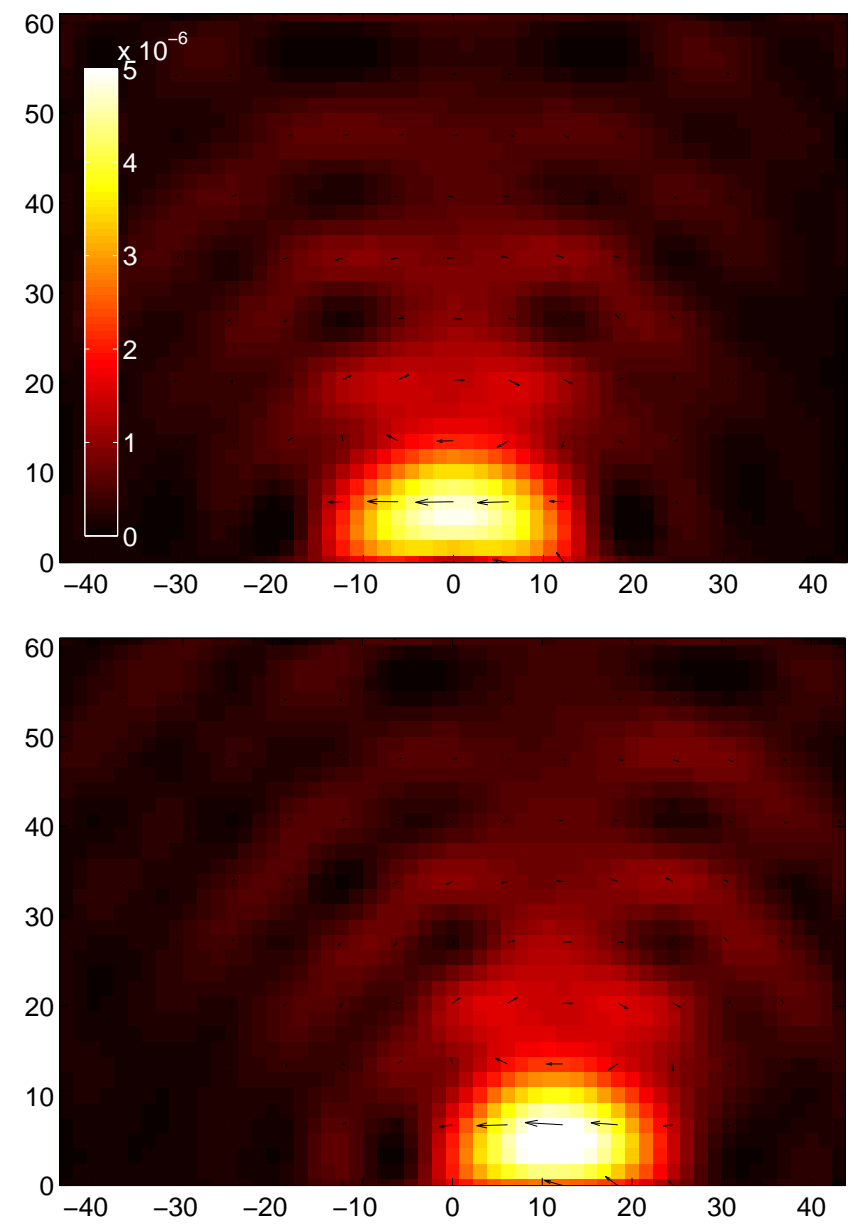

Fig. 2: Two examples of the electric-field distribution generated by means of a TREC when focusing over two adjacent positions centered at $0 \mathrm{~cm}$ and $12 \mathrm{~cm}$. The color scale represents the intensity of the electric field, while its orientation (polarization) is shown by arrows. Distances are in centimeters.

hardly affected by the displacement of the phase center. Fig. 2 shows that the focal spot, measured at half the peak intensity, is about a half-wavelength wide, as expected from diffraction theory [2]. Spatial resolution can be further improved by means of deconvolution techniques.

This procedure was repeated in order to generate focal spots scanning the device center across the $x$ direction, for an Efield oriented along the $x$ and $y$ axis. A probe was inserted into the device, by means of a small aperture opposed to the slot, in order to monitor the coupling of impinging wavefronts: in practice, this approach corresponds, e.g., to observing the voltage or current induced over a portion of a circuit. The results of this operation are shown in Fig. 3, keeping track of the peak intensity of the field recorded within the device and normalized to the peak value observed for the $x$-oriented wavefront field. These results prove that the proposed method can indeed image not only the position of the slot, but also

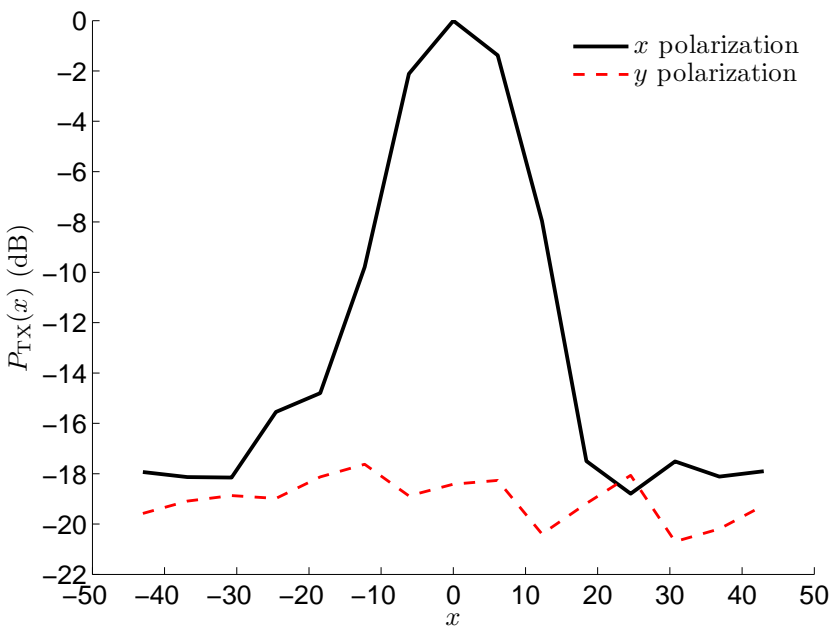

Fig. 3: Peak intensity of the signals transmitted at the interior of the EUT as a function of the focal-spot center, for two orientations of the electric field impinging over the device. The results are normalized to the value measured for a focalspot at $x=0$, for an electric field oriented along $x$.

its orientation, as witnessed by the $20 \mathrm{~dB}$ dynamic reduction observed in the coupling when switching between an $x$ - and $y$-oriented field.

\section{CONCLUSIONS}

We have shown that the TREC technique can be used in order to gain access to detailed information about the position and orientation of apertures in the shielding of a device. This approach thus appears to outperform anechoic and reverberation chambers in the identification of weak points in a device submitted to radiated interferences, of interest in EMC as well as hardening tests.

\section{REFERENCES}

[1] H. Moussa, A. Cozza, and M. Cauterman, "Directive wavefronts inside a time reversal electromagnetic chamber," in IEEE EMC Symposium, Austin, Texas, 2009., 2009.

[2] J. Stamnes, Waves in focal regions: propagation, diffraction, and focusing of light, sound, and water waves. Taylor \& Francis, 1986.

[3] A. Cozza, "Increasing peak-field generation efficiency of reverberation chamber," Electronics Letters, vol. 46, no. 1, pp. 38-39, 2010.

[4] A. Cozza and H. Moussa, "Enforcing deterministic polarisation in a reverberating environment," Electronics Letters, vol. 45, no. 25, pp. 12991301, 2009.

[5] A. Yariv, "Phase conjugate optics and real-time holography," IEEE Journal of Quantum Electronics, vol. 14, no. 9, pp. 650-660, 1978.

[6] A. Derode, A. Tourin, and M. Fink, "Random multiple scattering of ultrasound. II. Is time reversal a self-averaging process?" Physical Review $E$, vol. 64, no. 3, p. 36606, 2001.

[7] M. Fink, "Time reversal of ultrasonic fields. I. Basic principles," IEEE Transactions on Ultrasonics, Ferroelectrics and Frequency Control, vol. 39, no. 5, pp. 555-566, 1992.

[8] H. Moussa, A. Cozza, and M. Cauterman, "Experimental demonstration of directive pulsed wavefront generation in reverberation chambers," Electronics Letters, vol. 46, no. 9, pp. 623-624, 2010.

[9] K. Lee, EMP interaction: principles, techniques, and reference data: a handbook of technology from the EMP interaction notes. Taylor \& Francis, 1986. 